\title{
Comparación microscópica de la adaptación del cono maestro de gutapercha con conicidades $2 \%$ y $6 \%$
}

Montalván SS, Meneses A, Torres JP. Comparación microscópica de la adaptación del cono maestro de gutapercha con conicidades 2\% y 6\% Rev Estomatol Herediana 2005; 15 (2) : 107 - 111.

\section{Silvia Soledad Montalván Meza ${ }^{1}$ Abraham Meneses López ${ }^{2}$ John P. Torres Navarro ${ }^{3}$}

${ }^{1}$ Cirujano Dentista.

2Docente del Departamento Académico de Estomatología del Niño y del Adolescente.

${ }^{3}$ Docente del Departamento Académico de Clínica Estomatológica.

Facultad de Estomatología. Universidad Peruana Cayetano Heredia.

\section{Correspondencia}

Silvia Soledad Montalván Meza. Calle Puerto Príncipe 120 Urb. El Parral. Lima - 07, Perú

Teléfonos: $5361537 / 97190763$

e-mail: silvi_montalvan@ @otmail.com

\section{RESUMEN}

El propósito de este estudio, in vitro, fue analizar y comparar microscópicamente la adaptación de los conos de gutapercha, a nivel del tercio coronal, medio y apical, al ser obturados con la técnica de condensación lateral empleando conos maestros de gutapercha con conicidades .06 y .02 , en dientes preparados biomecánicamente con técnica híbrida utilizando los sistemas rotatorios PROTAPER y PROFILE. Se obturaron treinta premolares unirradiculares, 15 dientes por grupo, adicionalmente se registró el número de conos accesorios requeridos en cada obturación. Se fotografiaron los cortes transversales a través de un estereomicroscopio, para realizar un análisis métrico obteniendo áreas proporcionales de los espacios, en el programa AutoCad 2005. Los resultados se analizaron con la prueba t de Student; no se encontró diferencia estadísticamente significativa al comparar la adaptación de los conos de gutapercha entre el grupo I (conicidad ,02) y grupo II (conicidad ,06) $(\mathrm{p}>0,05)$; adicionalmente se observó el empleo de menor cantidad de conos accesorios al obturar con un cono maestro con conicidad , 06 considerándose como un hallazgo.

\section{Palabras clave: OBTURACIÓN DEL CONDUCTO RADICULAR / GUTAPERCHA}

Microscopic comparison of the adaptation of master gutta-percha $2 \%$ and $6 \%$ tapered cones.

ABSTRACT

The purpose of this in vitro study was to microscopically analyze and compare the adaptation of the gutta-percha cones, at the level of coronal third, middle and apical, when obturated with the lateral condensation technique using master gutta-percha cones with .06 and .02 tapers, in biomechanically prepared teeth with the hybrid technique using the PROTAPER and PROFILE rotational systems. Thirty premolar single-root were obturated, 15 teeth by group; additionally, the required number of cones in each obturation was registered. The stereomicroscopic crosssections were photographed in order to measure and compare the spaces in AutoCad 2005. The results, analyzed with Student's T test; did not find statistically significant difference when comparing the adaptation of the gutta-percha cones in group I (Taper .02) and group II (Taper .06) $(\mathrm{p}>0.05)$; additionally, the use of smaller amount of accessory cones was observed when obturating with a master cone with taper 06 , which in itself can be deemed a finding.

Keywords: ROOT CANAL OBTURATION / GUTTA-PERCHA

\section{Introducción}

La obturación del sistema de conductos tiene como objetivo principal el cierre hermético y el relleno tridimensional del conducto radicular (1-5). Al ocurrir una pobre adaptación de la gutapercha, las bacterias encuentran el espacio apropiado para desarrollarse y producir una lesión apical o mantener la lesión preexistente; este aspecto ha sido estudiado en diversos estudios donde relacionan una obturación deficiente y el fracaso endodóntico (6-8).

Desde hace mucho tiempo se usa la gutapercha para la obturar los conductos radiculares (9); por su facilidad de empleo y ser tolerado por el tejido periapical (10-13). En los últimos años el uso de las limas de níquel-titanio (NiTi) con conicidades mayores (al 4\% ó ,04 a más), han permitido conformar mejor los conductos; y para su obturación surgieron los conos de gutapercha con conicidades, 04 y ,06 mayores a las convencionales $(, 02)(14)$.

Existen varias técnicas de obturación, que con frecuencia se acompañan de afirmaciones mal fundamentadas sobre su mayor eficacia y menor filtración $(3,15)$; no obstante, la técnica de condensación lateral se enseña y practica en todo el mundo desde hace años (3), por lo sencillo y racional de su ejecución $(16,17)$. Una variación de esta técnica se introdujo en el programa predoctoral de la Universidad Ciencia y Salud de Oregon; donde se emplea un cono maestro con conicidad ,04 en lugar de uno convencional; siendo menor el espacio entre el cono y la pared del conducto, usando menos conos accesorios para obturarlo $(14,18,19)$.
La técnica de condensación lateral es una de las técnicas más aceptadas, y ha demostrado resultados clínicamente adecuados (20). Sin embargo se ha mencionado con frecuencia que dicha técnica no permite obtener una masa homogénea de gutapercha, tendiendo a formar espacios entre conos o entre los conos y pared del conducto, afectando la calidad de la obturación (20-23). Desde la introducción de los conos de gutapercha con mayor conicidad, se han realizado investigaciones para analizar su calidad de adaptación, al obturar con la técnica de condensación lateral, debido a los posibles vacíos en la obturación $(14,19,24,25)$.

Uno de los pasos que se tiene que cumplir antes de obturar, es la preparación biomecánica (PBM), este procedimiento consiste en la limpieza, desin- 
fección y conformación del conducto, proporcionando mayor diámetro a nivel cervical (10-12). Para mejorar dichos objetivos se fabricaron los sistemas rotatorios con limas de NiTi (26).

El óptimo ensanchamiento del diámetro apical es ideal (27), para lograr una mejor limpieza del conducto y facilitar la adaptación de la gutapercha (28). Por esta razón surgió el concepto de técnica híbrida, que es la combinación de diferentes sistemas de limas aprovechando sus mejores propiedades (27). En el año 2004 Walsch (27) clasifica la secuencia de sistemas que se puede emplear en la PBM, según la dificultad del caso y las ventajas que proporciona cada sistema. La conformación del conducto y el pre-ensanchamiento apical, se puede realizar con el sistema Protaper (Dentsply/Maillefer, Baillaigues, Switzerland), y el ensanchamiento apical con los diversos sistemas que se comercializan actualmente : K3 (SybronEndo, California), Profile (Dentsply Tulsa Dental), Hero (MicroMega SA, France) entre otros. Siguiendo este nuevo concepto y basándonos al estudio de Walsch, en el presente estudio se realizó la técnica híbrida, con los sistemas rotatorios Protaper y Profile, 06 .

Diversos estudios muestran que al obturar con la técnica de condensación lateral con conos de gutapercha convencionales, no se obtiene una masa uniforme de gutapercha por contener muchos conos accesorios. Con la introducción de conos de gutapercha con conicidades mayores, se abre una alternativa para mejorar la técnica. El propósito del estudio in vitro, fue comparar microscópicamente la adaptación del cono maestro de gutapercha con conicidad , 02 y, 06 ; al obturar con la técnica de condensación lateral, en dientes unirradiculares preparados biomecánicamente con técnica híbrida, utilizando los sistemas Protaper y Profile.

\section{Materiales y método}

Se utilizaron 30 premolares unirradiculares, conservados en solución fisiológica. La apertura cameral se realizó bajo refrigeración acuosa con fresas de diamante, continuando la preparación biomecánica bajo el concepto de técnica híbrida, con el Sistema rotacional Protaper, como indica el fabricante; la conductometría se determinó con la inserción de una lima $\mathrm{K}^{\circ} 15$ al conducto radicular hasta visualizarlo por el foramen apical, a la medida obtenida se le disminuyó un milímetro y se tomó radiografías. Posteriormente se instrumentó el tercio apical con el sistema Protaper hasta la lima F3 y con el sistema Profile, $06 \mathrm{~N}^{\circ} 35$ y 40 ; a cada cambio de lima se irrigó profusamente con solución de hipoclorito de sodio al 5,25\% (Clorox. Pe); al terminar la instrumentación los canales se llenaron con EDTA 17\%, (Biodinámica Quím. Ibipora, Bra.) para remover el barro dentinario, luego se procedió a sobrepasar con una lima $\mathrm{K} \mathrm{N}^{\circ} 10$ el foramen apical, y tras la irrigación final los conductos se secaron con conos de papel. Los dientes fueron distribuidos al azar en dos grupos de 15; y se obturó con la técnica de condensación lateral. En el grupo I se empleó un cono maestro con conicidad , $02 \mathrm{~N}^{\circ} 40$, con la ayuda de espaciadores se ingresaron conos accesorios; adicionalmente se registró el número de conos accesorios empleados en cada obturación. En el grupo II el procedimiento fue similar pero empleando un cono maestro con conicidad , $06 \mathrm{~N}^{\circ} 40$. Por ser el objetivo del estudio analizar la adaptación de los conos de gutapercha, no se empleó cemento en la obturación. Luego se procedió a cortar los conos a nivel de la cámara pulpar con un instrumento caliente y los accesos fueron obturados con policarboxilato de zinc. (Subiton Lab. San Fernando Arg.) Fueron numerados e incluidos en epoxi-resina transparente de curado rápido para cortarlos transversalmente a nivel del tercio coronal, medio y apical con un motor fijo y discos de diamante flexible con abundante refrigeración aire/agua. Los cortes se observaron mediante un estereomicroscopio (Leitz Wetzlar Germany) a $32 x$ de aumento $y$ se fotografiaron con una cámara digital (Coolpix 995 Nikon) 3.3MP. Las fotos se importaron al programa AutoCAD 2005 y se analizó la adaptación de la gutapercha de una manera cuantitativa, hallando la propor- ción de espacios, con respecto al área total del diámetro del conducto de las muestras.

Los resultados obtenidos se analizaron estadísticamente realizando un ANOVA para evaluar la homogeneidad de los valores obtenidos en ambas variables y para las variables numéricas se empleó el test $t$ de Student con un valor de significancia $\mathrm{p}<0,05$.

\section{Resultados y discusión}

El análisis estadístico de la adaptación de la gutapercha, en ambos grupos; muestra que en el grupo I a nivel del tercio coronal, el promedio del porcentaje del área de espacios entre conos y entre los conos y la pared del conducto radicular fue de $8,48 \%$; (Tabla 1) (Fig. 1) y en el grupo II de 6,47\%. (Tabla 2) (Fig.2)

Las imágenes obtenidas a este nivel, en ambos grupos fueron similares y se evidenció la presencia de espacios, si bien se observó en menor proporción en el grupo II; la diferencia no fue estadísticamente significativa ( $>0,05)$. (Tabla 3). Un estudio realizado por Zmener et al.(20) en el año 1998, observaron que en la técnica de condensa-

Tabla 1: Porcentaje promedio del total de espacios en el grupo I

\begin{tabular}{lll}
\hline & media & DS \\
\hline tercio coronal & 6,46 & 4,4 \\
tercio medio & 2,61 & 2,7 \\
tercio apical & 0,7 & 0,9 \\
\hline
\end{tabular}

Tabla 2: Porcentaje promedio del total de espacios en el grupo II

\begin{tabular}{lll}
\hline & media & DS \\
\hline tercio coronal & 8,48 & 3,2 \\
tercio medio & 4,52 & 5,2 \\
tercio apical & 1,59 & 3,3 \\
\hline
\end{tabular}

Tabla 3: Comparación de los porcentajes del total de espacios presentes entre el grupo I y grupo II

\begin{tabular}{llll} 
& $\begin{array}{l}\text { grupo I } \\
\text { media }\end{array}$ & $\begin{array}{l}\text { grupo II } \\
\text { media }\end{array}$ & $\mathrm{p}$ \\
\hline tercio coronal & 8,48 & 6,47 & 0,159 \\
tercio medio & 4,52 & 2,61 & 0,215 \\
tercio apical & 1,59 & 0,37 & 0,191 \\
\hline
\end{tabular}


ción lateral a nivel del tercio coronal y en el tercio medio, los conos de gutapercha no mostraban coalescen-cia entre sí, existiendo presencia de espacios entre los conos y entre los conos y la pared del conducto, pero a pesar de todo, se mostró una tendencia clara de los conos de gutapercha a adaptarse y reproducir las irregularidades de las paredes del conducto preparado. Dichas situaciones también se observaron en ambos grupos muestrales del estudio que se realizó.

A nivel del tercio medio en el grupo I, el promedio del porcentaje del área de los espacios fue de 4,52\%; (Tabla 1) (Fig. 3) y en el grupo II de 2,61\% (Tabla 2) (Fig. 4)

En el análisis de las imágenes en este tercio, no se encontró diferencia estadística significativa $(\mathrm{p}>0,05)$, sin embargo se observó que en el grupo II, la mayoría de los casos presentó cono

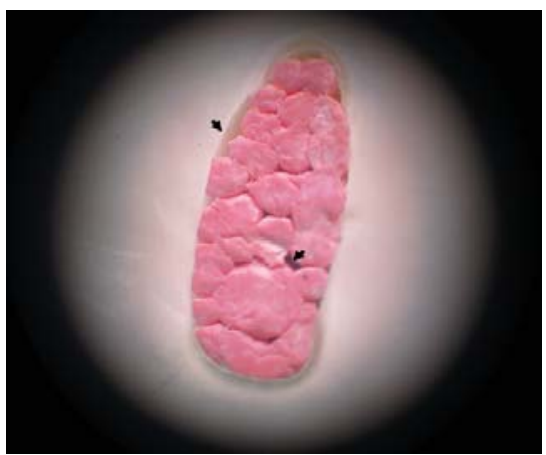

Fig. 1. Microfotografía del tercio coronal del grupo I (obturado con cono maestro con conicidad, 02) a $32 \mathrm{X}$. Se observa presencia de espacios entre conos y entre los conos y las paredes del conducto radicular (flechas).

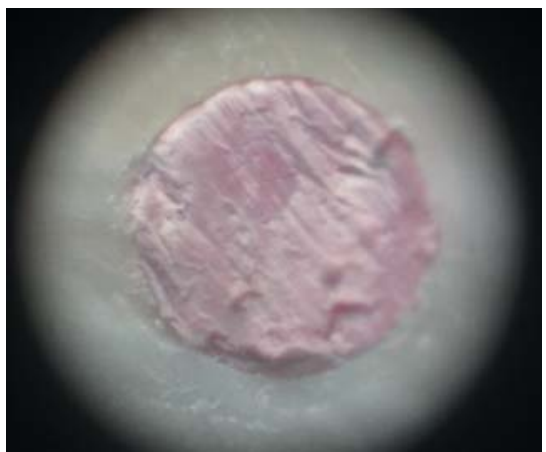

Fig. 4. Microfotografía del tercio medio de grupo II a $64 X$. Se observa la presencia de cono único en toda el área del conducto radicular. único; lo cuál no ocurrió en las muestras del grupo I, donde se observó una imagen similar al del tercio coronal, con presencia de varios conos accesorios, y espacios. (Tabla 3). Dicho hallazgo, también fue encontrado en el estudio que realizaron Hembrough et al.(24) en el año 2002, donde evaluaron tres conos maestros de gutapercha (con conicidad ,02,054 y, 06) empleando la técnica de condensación lateral; a nivel del tercio medio, observaron que en los grupos que emplearon los conos con conicidades, 054 y, 06 presentaron en la mayoría de casos, sólo cono único, mientras que en el grupo que contenía el cono maestro con conicidad, 02 presentaba varios conos de gutapercha accesorios y espacios entre ellos. Estos resultados se pueden atribuir a que la conformación final del conducto radicular, después de preparar con la técnica híbrida, empleando una lima Profile .06 como

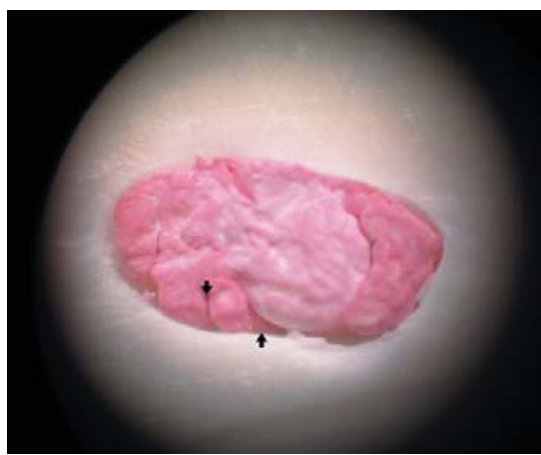

Fig. 2. Microfotografía del tercio coronal del grupo II (obturado con cono maestro con conicidad ,06) a $32 X$. Se observa presencia de espacios entre conos y entre los conos y las paredes del conducto radicular (flechas).

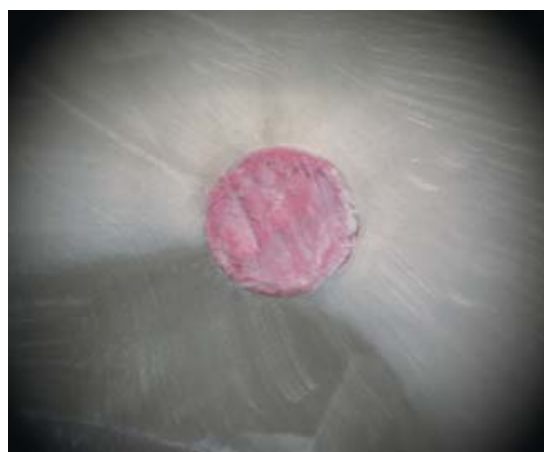

Fig. 5. Microfotografía del tercio apical del grupo I a $64 \mathrm{X}$. Se observa la presencia de cono único en toda el área del conducto radicular. acabado final, la conicidad obtenida sería equivalente al de los conos maestros con conicidad .06 del grupo II, y a nivel del tercio medio se tendría en la mayoría de las muestras un diámetro similar entre el conducto preparado y el cono maestro empleado. En contraste al grupo I, donde el cono maestro empleado tiene una conicidad, 02 teniendo un diámetro más pequeño con respecto al espacio del canal preparado, $\mathrm{y}$ por consiguiente el espacio es suficiente para la penetración del espaciador y la inserción de varios conos accesorios para completar la obturación.

Por último a nivel del tercio apical, en el grupo I, el promedio del porcentaje del área de los espacios encontrados a este nivel es de 1,59\%; (Tabla 1) (Fig. 5) y en el grupo II es de $0,037 \%$. (Tabla 2) (Fig.6).

Analizando los grupos I y II, a nivel del tercio apical, no se encontró dife-

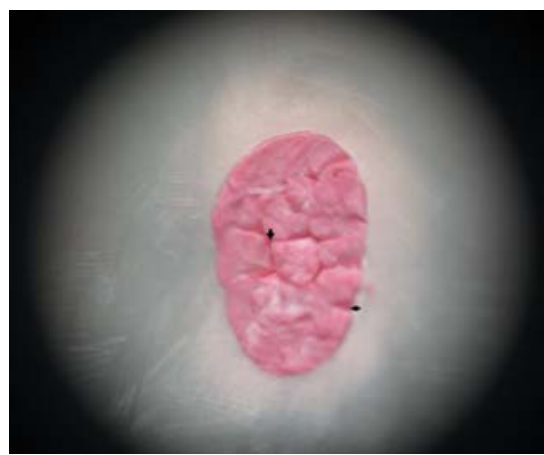

Fig. 3. Microfotografía del tercio medio del grupo I a $32 \mathrm{X}$. Se observa presencia de espacios entre conos y entre los conos y las paredes del conducto radicular (flechas).

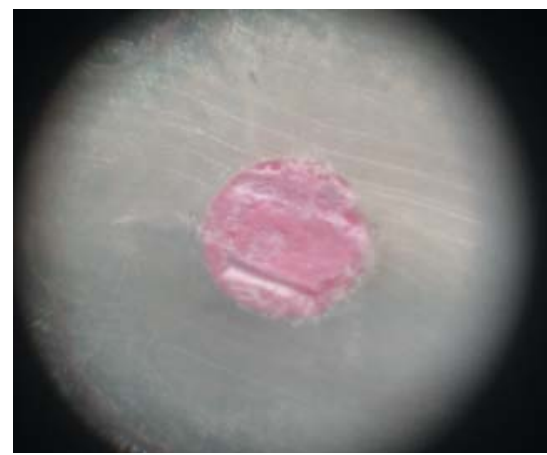

Fig. 6. Microfotografía del tercio apical de grupo II a 64X. Se observa la presencia de cono único en toda el área del conducto radicular. 
rencia estadísticamente significativa ( $>0,05)$. Las imágenes que se observó en la mayoría de los casos en ambos grupos fue cono único. (Tabla 3). En otro estudio de Zemener et al. (21) en el año 2000, después de analizar la calidad de la obturación ente las técnicas de condensación lateral, y dos técnicas de gutapercha termoplastificadas en premolares unirradiculares, apreció a nivel del tercio apical una conformación netamente circular que llevó a una buena adaptación de la gutapercha evidenciándose conos únicos a este nivel. Dicho aspecto justifica lo observado a este nivel, en el grupo I y II en el estudio realizado.

En general, la morfología del conducto de los premolares, no afectó en los resultados de la adaptación de los conos de gutapercha a las paredes del conducto radicular, debido a que en la mayoría de los casos, la obturación abarcó dichas irregularidades del conducto y a nivel de los tercios comparados entre el grupo I y el grupo II, las diferencias estadísticas encontradas no fueron significativas. ( $>>0,05)$. (Tabla 3 )

Adicionalmente se realizó el conteo de conos accesorios empleados en cada obturación, donde se observó que en los dientes obturados con un cono maestro con conicidad, 02 se empleó un promedio de seis conos accesorios más que al obturar con un cono maestro con conicidad ,02. Este hallazgo es analizado en la investigación realizado por Hembrough et al. (24) en el año 2002, al evaluar la calidad de adaptación y la eficacia de la técnica de condensación lateral empleando tres conos maestros con conicidad , 02,054 y , 06; los resultados no mostraron diferencias estadísticamente significativas al evaluar la calidad de la adaptación de la obturación. Sin embargo al evaluar la eficiencia en la obturación era perceptiblemente mayor con los conos maestros con conicidades $, 054 \mathrm{y}, 06$ que con un cono maestro de conicidad , 02 debido a que en los dos primeros grupos se emplearon menor cantidad de conos accesorios comparando con el grupo que se obturó con un cono maestro con conicidad, 02 .
El empleo de conos de gutapercha con conicidad aumentada como cono maestro para mejorar la obturación de los conductos preparados con limas con conicidades mayores, se justifica como una variación de la técnica de obturación de condensación lateral, debido a los estudios realizados en estos últimos años. Si bien es poca la literatura que existe actualmente por ser un material nuevo en el mercado, dichos estudios han demostrando su buena adaptación y eficiencia en la obturación $(18,19,24)$.

\section{Agradecimientos}

A la Blga. Luz Marina Pacheco del Departamento de Microbiología de la Facultad de Ciencias de la Universidad Peruana Cayetano Heredia, por las facilidades prestadas para la realización de la presente investigación.

Al Lcdo. Juvenal Castromontes y Alonso Tenorio, del Departamento de Física y Matemática de la Facultad de Ciencias de la Universidad Peruana Cayetano Heredia por su colaboración en el análisis métrico del estudio.

\section{Referencias bibliográficas}

1. Ingle J, Beveridge E. Endodoncia. 3a ed. México: Nueva Editorial Interamericana;1987.

2. Weine F. Endodontic Therapy. 4th ed. St Louis: CV Mosby Co; 1989.

3. Harty FP. Endodoncia en la práctica clínica. 4a ed. Mexico: McGraw-Hill; 1999.

4. Schilder H. Filling root canals in three dimensions. Dent Clin North Am 1967; 11:723-44.

5. Lacobelli L, Lanniciello L, Catalano C, Rupe A. Observación de seis métodos de obturación de conductos radiculares con el estereomicroscopio. Compendio 1993/1994; 9(3).

6. Walton R, Torabinejad M. Principles and practice of endodontics. 2nd ed. Mexico: Mc Graw-Hill Interamericana; 1998.

7. Ingle JI. A standardized endodontic technique utilizing newly designed instruments and filling materials. Oral Surg Oral Med Oral Pathol 1961; 14:83-91.
8. Grossman L, Shepard L, Pearson L. Roentgenologia and clinical evolution of endodonticali treated teeth. Oral Surg Oral Med Oral Pathol Oral Radiol Endod 1964; 17(3): 368-73.

9. Goldberg F. La obturación del conducto radicular: nuevos materiales y técnicas. Rev Aten Argent Odontol 1996;35:5-9

10. Leonardo-Leal Endodoncia, tratamiento de los conductos radiculares. 2da ed. Buenos Aires: Editorial Médica Panamericana; 1994.

11. Villena H. Terapia Pulpar, Lima: Editorial Universidad Peruana Cayetano Heredia, Facultad de Estomatología; 2001.

12. Cohen S. Burns R. Vías de la pulpa. 7a ed. Madrid: Harcourt; 1999.

13. Weine F. Un análisis preliminar de los materiales de obturación de conducto del siglo 21. Compendio. 1993/1994; 9(1):41-50.

14. Baumgartner JC, Tinkle JS. Sophomore endodontic laboratory manual. Portland: Oregon Health \& Science University; 2002.

15. Brayton SM, Davis SR, Goldman M. Gutta-percha root canal fillings. An in vitro analysis. I. Oral Surg Oral Med Oral Pathol 1973; 35(2):226-31.

16. Lasala A. Endodoncia. 8a ed. Barcelona: Salvat Editores SA; 1998.

17. Glickman GN, Gutmann JL. Contemporary perspectives on canal obturation. Dent Clin North Am 1992; 36(2):327-41.

18. Wilson BL, Baumgartner JC. Comparison of spreader penetration during lateral compaction of .04 and .02 tapered gutta-percha. J Endodon 2003; 29(12):828-31

19. Bal AS, Hicks ML, Barnett F. Comparison of laterally condensed .06 and .02 tapered gutta-percha and sealer in vitro. J Endod 2001; 27(12):786-8.

20. Zmener O, Campuzano A. Análisis de la adaptación de la gutapercha termoplastificada del sistema Thermafil a las paredes del conduc-to radicular. Un estudio in vitro. Rev Asoc Odontol Argent 1998; 86(1):26-9.

21. Zmener O, Perruchino R, Zacarías M. Análisis de la calidad de la obturación endodóntica obtenida 
por medio de dos técnicas de gutapercha termoplastificada. Endodoncia 2000; 18(1):16-21.

22. Bowman CJ, Baumgartner JC. Guttapercha obturation of lateral grooves and depressions. J Endod 2002; 28(3):220-3.

23. Eguchi DS, Peters DD, Hollinger JO, Lorton L. A comparison of the area of the canal space occupied by gutta-percha following four guttapercha obturation techniques using procosol sealer. J Endod 1985;
11(4):166-75.

24. Hembrough MW, Steiman HR, Belanger KK. Lateral condensation in canals prepared with nickel titanium rotary instruments: an evaluation of the use of three different master cones. J Endod 2002; 28(7):516-9.

25. Lea CS, Apicella MJ, Mines P, Yancich PP, Parker MH. Comparison of the obturation density of cold lateral compaction versus warm vertical compaction using the conti- nuous wave of condensation technique. J Endod 2005; 31(1):37-9.

26. Spangberg L. The wonderful world of rotary root canal preparation. Oral Surg Oral Med Oral Pathol Oral Radiol Endod 2001; 92(5):479.

27. Walsch H. The hybrid concept of nickel-titanium rotary instrumentation. Dent Clin North Am. 2004 Jan;48(1):183-202.

28. Mounce RE. The K3 rotary nickeltitanium file system. Dent Clin North Am 2004; 48(1):137-57. 